\title{
Dynamics of solitonlike pulse propagation in birefringent optical fibers
}

\author{
Nail Akhmediev \\ Optical Sciences Centre, Institute of Advanced Studies, The Australian National University, \\ Canberra, Australian Capital Territory 0200, Australia \\ J. M. Soto-Crespo \\ Instituto de Óptica, Consejo Superior de Investigaciones Científicas, Serrano 121, 28006 Madrid, Spain
}

(Received 1 February 1994)

\begin{abstract}
Integrated Stokes parameters are used to describe solitonlike pulse propagation and its state of polarization in birefringent optical fibers. A qualitative analysis on the Poincare sphere is developed to describe the evolution of the state of polarization of the soliton as a whole. Simple analytic equations describing the pulse evolution are derived in the approximation of the average profile. It is shown that two qualitatively different regimes of propagation are possible, depending on the material parameters and on the initial conditions. The approach takes into account the radiation processes. The results of the analytical approach are compared with exact numerical calculations.
\end{abstract}

PACS number(s): 42.81.Dp, 42.81.Gs

\section{INTRODUCTION}

The propagation of solitonlike pulses in birefringent optical fibers has been the subject of intensive investigations during recent years [1-10]. Birefringence in optical fibers can be induced deliberately or can be residual due to imperfections in the drawing process. In both cases the birefringence must be taken into account when considering pulse propagation along the fiber. It was shown by Evangelides et al. [3] that the same state of polarization applies to the soliton as a whole. The averaging of the residual birefringence, due to random variation in the principal axes, has also been considered in [3] using the Stokes-parameter formalism. In this paper we are interested in the details of pulse propagation in regular polarization-maintaining fibers to clear up basic processes of propagation.

The Stokes-parameter formalism was first shown to be an appropriate method to study the nonlinear two-core fiber by Daino, Gregory, and Wabnitz [11]. They considered the coupler response to continuous waves (cw) when the dynamical system has a finite number of degrees of freedom. Then the classical theory of dynamical systems can be applied to the problem to make qualitative analysis of solutions on a two-dimensional surface (Poincaré sphere). The use of the Stokes parameters to study the propagation of solitons in fibers has been considered by Evangelides et al. [3]. In the case of pulse propagation, the dynamical system has an infinite number of degrees of freedom, and full analysis has to be done in an infinite-dimensional phase space. However, in the case of solitonlike pulses, the approximation of the average profile can be applied to the problem. We show that, in this special approximation, an equation for the differential soliton Stokes parameters can be reduced to equations for integrated Stokes parameters, and the whole problem can be reduced to a dynamical system with a finite number of degrees of freedom. This allows us to consider solutions of the system as trajectories on the Poincaré sphere.

The approximation of the average profile is a consequence of the Hamiltonian structure of the initial equations. In fact, the effect of the fiber birefringence can be considered as a Hamiltonian perturbation to a single nonlinear Schrödinger equation (NLSE). This means that, in the first place, solitons are robust relative to this perturbation, and that the changes birefringence causes to the pulse do not destroy the soliton itself [12]. Therefore, we can consider solitonlike pulses as perturbed solitons of a single NLSE, as they only change slowly upon propagation. The modifications that the pulses suffer on propagation are due to energy transfer taking place between each linearly polarized mode. The pulse profile itself is roughly defined by the NLSE. The changes of the polarization state of the soliton are the most important effects in this problem. The polarization state of the pulse as a whole can be described by integrated values which change slowly in the course of soliton propagation along the fiber.

Pulse propagation in optical fibers is mainly governed by two parameters: the linear beat length between modes and the nonlinear beat length. Another independent parameter is the input pulse width. Up to now the exact expression for the nonlinear beat length was unclear although it had been shown by Blow, Doran, and Wood [2] that it plays an essential role in the dynamics of soliton propagation in birefringent fibers. In this work we derive an analytic expression for this important physical parameter in terms of the ratio between cross- and self-phase modulation and the soliton period. The pulse can propagate in different regimes which depend on the relative values of these three parameters. The combined parameter which we call the "evolution parameter" is related to the Hamiltonian and governs the regime of propagation. The aim of this paper is to investigate these regimes qualitatively using the integrated Stokes parameters which characterize the state of polarization of the pulse as a 
whole. The results of this analysis are presented as closed trajectories on the Poincaré sphere.

Another important effect which we take into account in our analysis is the radiation of small amplitude waves by solitons during the propagation along the fiber. Radiation emission is a general property of nonintegrable systems, and it accompanies the solitonlike pulse propagation. One of its origins resides in the higher order dispersion of the fiber [13]. The radiation originated in this way (which can be described by the theory "beyond all orders" [14]) is usually negligible and can be ignored. Another reason for radiation is the oscillatory behavior of the solitonlike pulse itself. The intensity of this radiation is much higher, and it depends on the amplitude of the oscillations. Therefore, although the dynamical system which we describe is Hamiltonian, there could be losses of energy from the leading pulse. This effect influences the propagation dynamics of the pulse in general and its state of polarization in particular. The influence of the radiation is also described qualitatively in our theory.

If the initial conditions are not solitons but pulses of a general form, they are either dispersed on propagation (if the pulse amplitude is smaller than half of that corresponding to the fundamental soliton [15]) or broken up, leaving most of the energy in a few highly compressed single solitons if the initial signal is a multisoliton pulse [2]. Dispersed radiation does not carry information and can be ignored. In the case of a multisoliton pulse, our analysis is applicable to each solitonlike pulse produced after the breakup of a multisoliton pulse. For simplicity we assume that the pulses are well separated.

\section{STATEMENT OF THE PROBLEM}

Pulse propagation in a birefringent optical fiber can be described in terms of two coupled nonlinear Schrödinger equations. In a reference frame traveling along the $z$ axis with the common group velocity, this set of equations is of the form [3]

$$
\begin{aligned}
& i U_{\xi}+\beta U+\frac{1}{2} U_{\tau \tau}+\left(|U|^{2}+A|V|^{2}\right) U+(1-A) V^{2} U^{*}=0, \\
& i V_{\xi}-\beta V+\frac{1}{2} V_{\tau \tau}+\left(A|U|^{2}+|V|^{2}\right) V+(1-A) U^{2} V^{*}=0,
\end{aligned}
$$

where $U$ and $V$ are the slowly varying envelopes of the two linearly polarized components of the field along the $x$ and $y$ axes, $\beta$ is half the difference between the propagation constants, $A$ is the normalized ratio of the nonlinear susceptibilities, $\xi$ is the normalized longitudinal coordinate, $\tau$ is the normalized retarded time, and the asterisk denotes complex conjugate. We ignore in (1) the difference between the group velocities of each component. This can be done for optical pulse durations in the picosecond range and for relatively small linear birefringences [6]. The set of equations (1) has been studied extensively in plasma theory. Some exact results are presented in $[16,17]$. The particular case for $A=1, \beta=0$ is integrable (using the inverse scattering method [18]).

The set of equations (1) has at least two integrals, the action (total energy of the pulse)

$$
Q=\int_{-\infty}^{\infty}\left(|U|^{2}+|V|^{2}\right) d \tau
$$

and the Hamiltonian

$$
\begin{aligned}
H=\int_{-\infty}^{\infty} & \left\{-\beta\left(|U|^{2}-|V|^{2}\right)+\frac{1}{2}\left(\left|U_{\tau}\right|^{2}+\left|V_{\tau}\right|^{2}\right)\right. \\
& -\frac{1}{2}\left(|U|^{4}+|V|^{4}\right)-A|U|^{2}|V|^{2} \\
& \left.-\frac{1}{2}(1-A)\left(U^{2} V^{* 2}+U^{* 2} V^{2}\right)\right\} d \tau .
\end{aligned}
$$

Equations (6) can now be written in a canonical form $[19,20]$,

$$
i U_{\xi}=\frac{\delta H}{\delta U^{*}}, i V_{\xi}=\frac{\delta H}{\delta V^{*}} .
$$

Equations (3) and (4) define a Hamiltonian dynamical system on an infinite-dimensional phase space of two complex functions $U, V$ which decrease to zero at infinity and can be analyzed based on the theory of Hamiltonian systems. This means that the behavior of the solutions is defined to a large extent by a singular points of the system [stationary solutions of Eqs. (6) below] and depends on the type of these points (as determined by the stability of its stationary solutions).

We are interested in pulselike solutions of (1) which are close to the soliton solutions of a single NLSE. By representing the field components in the form

$$
U=u(\xi, \tau, q) e^{i q \xi}, \quad V=v(\xi, \tau, q) e^{i q \xi},
$$

Eqs. (1) become

$$
\begin{aligned}
& i u_{\xi}-(q-\beta) u+\frac{1}{2} u_{\tau \tau}+\left(|u|^{2}+A|v|^{2}\right) u \\
& +(1-A) v^{2} u^{*}=0, \\
& i v_{\xi}-(q+\beta) v+\frac{1}{2} v_{\tau \tau}+\left(A|u|^{2}+|v|^{2}\right) v \\
& +(1-A) u^{2} v^{*}=0,
\end{aligned}
$$

where $q$ is the soliton parameter, $2 \pi / q$ is proportional to the soliton period, and $2 \sqrt{2 q}$ is proportional to the energy of a soliton.

Equations (6) have two simple stationary solutions, viz., linearly polarized soliton waves along the slow axis,

$$
u=\frac{\sqrt{2(q-\beta)}}{\cosh [\sqrt{2(q-\beta)} \tau]}, v=0
$$

and linearly polarized soliton waves along the fast axis,

$$
u=0, \quad v=\frac{\sqrt{2(q+\beta)}}{\cosh [\sqrt{2(q+\beta)} \tau]} .
$$

In the absence of linear birefringence $(\beta=0)$ or nonlinear birefringence $(A=1)$, the pulses (7) and (8) degenerate into a soliton of a single NLSE,

$$
\sqrt{u^{2}+v^{2}}=\frac{\sqrt{2 q}}{\cosh (\sqrt{2 q} \tau)} .
$$

This solution can be linearly polarized along any direction in the $(u, v)$ plane.

The solutions (7) and (8) can be conveniently represented on the energy-dispersion diagram [see Fig. 1(a)]. The energy $Q$ on this diagram is given by Eq. (2). The energy of the NLSE soliton (9), $Q$ versus $q$, is shown by the 

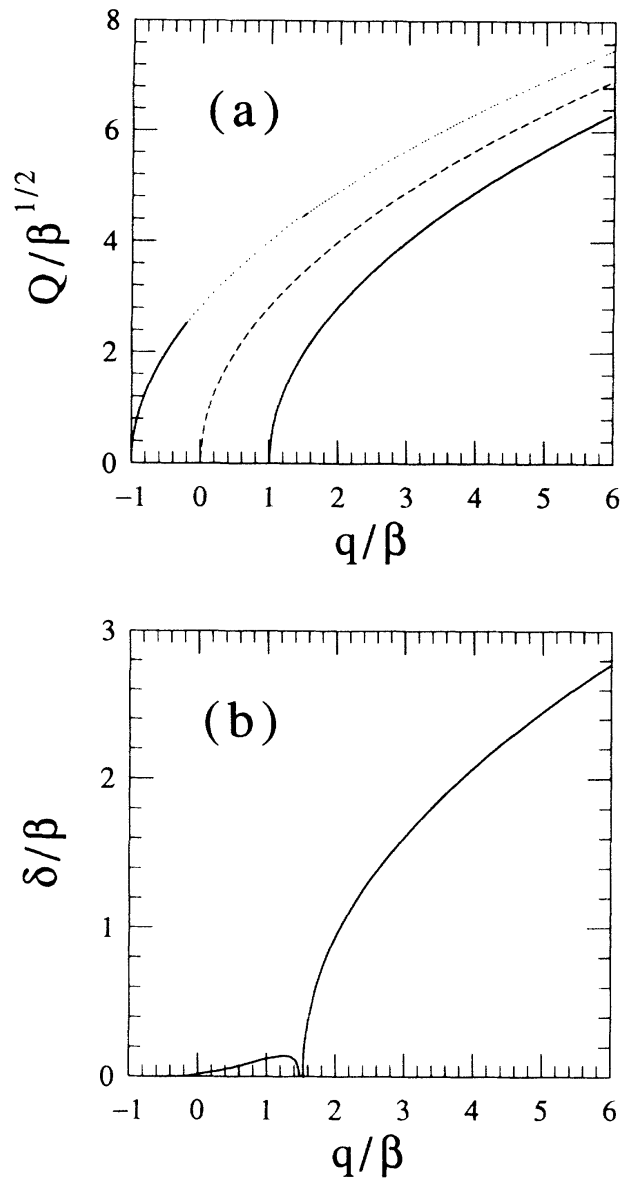

FIG. 1. (a) Energy dispersion diagram for linearly polarized solitons. (b) Growth rates for the fast linearly polarized solitons.

dashed line. Intuitively, we can say that any input pulse, whose propagation is governed by the perturbed NLSE, evolves in such a way that its energy stays in the interval defined by the stripe between the top and lowest lines in Fig. 1(a), provided that the initial conditions are solitonlike pulses. This stripe is the area of allowed motion for these pulses in this dynamical system. Any given solitonlike pulse can be shown on this diagram by its representative point which specifies the energy and the $q$ parameter of the solution. Note that the solution does not necessarily have to be a stationary one.

The linearly polarized solitons along the fast axis (upper branch in this diagram) are known to be unstable in a certain range of parameters [2]. We have numerically calculated the perturbation growth rates at $A=\frac{2}{3}$ using the technique described in [22]. The results are represented in Fig. 1(b). The perturbation with the largest growth rate has a real eigenvalue for $q / \beta>1.53$ and has a complex eigenvalue for $-0.2<q / \beta<1.475$ [In Fig. 1 (b) we represent only its real part]. For the values of $q / \beta$ outside these two intervals, the fast waves are stable, or at least their perturbation growth rates are too small to be found by our numerical technique. There are other stationary soliton states above the upper curve [21] but they are beyond the scope of this particular paper.
The linearly polarized solitons along the slow axis (lower branch in this diagram) are known to be stable [2] at least in the interval $0<A<1$. This is also intuitively expected because there are no other stationary soliton states below this curve at $(0<A<1)$ to which they could converge during propagation.

A convenient way to solve the above equations is to use the Stokes parameters [3], which are defined by

$$
\begin{aligned}
& s_{0}=|u|^{2}+|v|^{2}, \\
& s_{1}=|u|^{2}-|v|^{2}, \\
& s_{2}=u^{*} v+u v^{*}, \\
& s_{3}=-i\left(u^{*} v-u v^{*}\right) .
\end{aligned}
$$

All these four parameters are real functions of $\xi$ and $\tau$. They vary along the fiber as well as across the pulses. Hence we call them differential Stokes parameters. Using them and taking into account the fact that all the fields must decay to zero at infinity, Eqs. (6) can be written in the form

$\frac{d}{d \xi} \int_{-\infty}^{\infty} s_{0} d \tau=0$,

$\frac{d}{d \xi} \int_{-\infty}^{\infty} s_{1} d \tau=-2(1-A) \int_{-\infty}^{\infty} s_{2} s_{3} d \tau$,

$\frac{d}{d \xi} \int_{-\infty}^{\infty} s_{2} d \tau=2 \beta \int_{-\infty}^{\infty} s_{3} d \tau+2(1-A) \int_{-\infty}^{\infty} s_{1} s_{3} d \tau$,

$\frac{d}{d \xi} \int_{-\infty}^{\infty} s_{3} d \tau=-2 \beta \int_{-\infty}^{\infty} s_{2} d \tau$,

where the dependence on $\tau$ has been eliminated due to the integration, and all the magnitudes are real ones.

Equations (11) are integrodifferential equations which can be even more difficult to solve than the original set. However, if we are interested in integrated values $S_{i}=\int_{-\infty}^{\infty} s_{i} d \tau$, then these equations can be simplified. Moreover, if we are interested in soliton propagation, then the integrated values provide most of the information we need in the problem. The first equation, for example, is nothing other than the conservation of the total energy of the solitary pulse. The third and the fourth equations in (11) show that the integrated values $\int_{-\infty}^{\infty} s_{2} d \tau$ and $\int_{-\infty}^{\infty} s_{3} d \tau$ are rotating around the axis $s_{1}$ with frequency $2 \beta$. However, the integrals of the products $s_{2} s_{3}$ on the right-hand side of these equations make the problem quite complicated to solve. To make a first step towards solving this generally complicated problem, we shall use an approximation which is suggested by the exact numerical solution of Eqs. (6) for several solitonlike pulse initial conditions.

\section{APPROXIMATION OF THE AVERAGE PROFILES}

We are interested in the problem of solitonlike pulse propagation. That is, we shall assume that the shape of each component of the pulses hardly changes upon propagation, and that the phase chirp across each pulse is negligible. However, the amplitudes can change with the distance $\xi$ because of the energy transfer taking place be- 
tween the two components of the field. Obviously, the actual shape oscillates around the considered average profile. However, these oscillations should be small: in nonintegrable dynamical systems any field oscillation generates small amplitude waves around the soliton which are radiated away. Their intensity is proportional to the amplitude of these oscillations. The energy of the soliton would decrease in this case with the distance $\xi$.

As we shall see in what follows, this assumption is a good approximation when the linear birefringence $\beta$ is smaller than the soliton parameter $q$. More precisely, the linear beat length $L_{l} \approx \pi / \beta$ should be larger than the nonlinear beat length $L_{\mathrm{nl}}$ which we shall define later. We shall also show that this assumption allows us to qualitatively describe soliton propagation even when $L_{l}$ is comparable to or less than $L_{\mathrm{nl}}$. Then, in a first approximation, we shall assume that the solution is separable in the following way:

$$
u=X(\xi) f(\tau), \quad v=Y(\xi) f(\tau),
$$

where $f(\tau)$ is a real function defining the common average (constant) profiles and $X(\xi)$ and $Y(\xi)$ are complex amplitudes. The approximation can be improved greatly by adding new terms in (12), but this is beyond the scope of this paper. Equations (11) become

$$
\begin{aligned}
& \frac{d}{d \xi} S_{0}=0, \\
& \frac{d}{d \xi} S_{1}=-2 g S_{2} S_{3}, \\
& \frac{d}{d \xi} S_{2}=2 \beta S_{3}+2 g S_{1} S_{3}, \\
& \frac{d}{d \xi} S_{3}=-2 \beta S_{2},
\end{aligned}
$$

where the integrated Stokes parameters divided by the integral $\int_{-\infty}^{\infty} f^{2} d \tau$ are given by

$$
\begin{aligned}
& S_{0}=\left(|X|^{2}+|Y|^{2}\right), \\
& S_{1}=\left(|X|^{2}-|Y|^{2}\right), \\
& S_{2}=\left(X^{*} Y+X Y^{*}\right), \\
& S_{3}=-i\left(X^{*} Y-X Y^{*}\right),
\end{aligned}
$$

and $g$, the nonlinear birefringent coefficient, is defined by

$$
g=(1-A) \frac{\int_{-\infty}^{\infty} f^{4} d \tau}{\int_{-\infty}^{\infty} f^{2} d \tau} .
$$

The nonlinear beat length $L_{\mathrm{nl}}$ is proportional to this coefficient: $L_{\mathrm{nl}} \approx \pi / g$. The value of $g$ depends on the soliton parameter $q$ as well as on $A$. To estimate roughly the dependence on $q$ we calculate $g$ using the average pulse shape (9). In this case $g=\frac{4}{3} q(1-A)$. The deviation of this value from the average of that corresponding to the slow and fast linearly polarized solitons is $\Delta g=\frac{4}{3} \beta(1-A)$, which is small at small $\beta$. Then $g$ is, roughly speaking, proportional to $q$ and changes its sign at $A=1$. It is remarkable that $g$ becomes zero when $A=1$.
Equations (13) can be written in a vector form:

$$
\frac{d}{d \xi} \mathbf{S}=2 \beta\left[\mathbf{e}_{1} \times \mathbf{S}\right]+2 g S_{3}\left[\mathbf{e}_{3} \times \mathbf{S}\right] \text {, }
$$

where $\mathbf{S}=\left(S_{1}, S_{2}, S_{3}\right)$ is the Stokes vector in a threedimensional space, $e_{1}$ and $e_{3}$ are unit vectors along the axes 1 and 3, respectively, and $X$ indicates vector product. Equation (16) describes a double rotation around the axes 1 and 3 , respectively.

The set (13) has an integral of motion

$$
S_{1}^{2}+S_{2}^{2}+S_{3}^{2}=S_{0}^{2} \text {, }
$$

which is a consequence of the energy conservation (2), and indicates that, within the approximation we are making, the evolution of any solution can be qualitatively analyzed as a motion of the Stokes vector $\mathbf{S}$ on the Poincare sphere. The set (13) has a second integral

$$
W=\frac{g}{2 \beta} S_{3}^{2}-S_{1},
$$

which is a consequence of the conservation of the Hamiltonian (3). In our approximation

$$
\begin{aligned}
W=\frac{H}{\beta I}+ & \frac{g S_{0}^{2}}{2 \beta(1-A)}-\frac{\rho S_{0}}{2 \beta}, \\
& \text { where } \rho=\frac{\int_{-\infty}^{\infty} f_{\tau}^{2} d \tau}{\int_{-\infty}^{\infty} f^{2} d \tau}, I=\int_{-\infty}^{\infty} f^{2} d \tau .
\end{aligned}
$$

Different values of $W$ correspond to different regimes of soliton propagation (i.e., different trajectories on the Poincaré sphere). From now on, we shall call it the evolution parameter. The convenience of the evolution parameter (in contrast to the Hamiltonian) is that it is constant along the energy-dispersion curves for fast and slow solitons in Fig. 1(a). We can conclude preliminarily that the same value of $W$ corresponds to the same type of solution for different soliton parameters $q$.

Let us choose as initial conditions one of the linearly polarized solitons given by Eqs. (7) or (8). We can consider now that initially $X(\xi=0=1, Y(\xi=0)=0$, or vice versa, and therefore the integrated Stokes parameters in (14) are normalized so that $S_{0}(\xi=0)=1$. In principle, the value of $S_{0}$ is conserved, as the first of Eqs. (14) proves. However, if the pulse changes its shape, and if it radiates energy as a result of this reshaping, then $S_{0}$ can, and usually does, decrease during propagation. For small perturbations of the NLSE these changes are slow $\left(d S_{0} / d \xi \ll S_{0}\right)$ and can be considered adiabatic. The second integral also depends on the energy of the pulse, and decreases if energy radiation takes place. We will assume these processes are slow and ignore them to a first approximation.

Before proceeding with the general theory, let us first consider two limiting cases.

\section{A. Special cases: (a) $\beta=0$, and (b) $A=1$}

(a) In Eqs. (6), $\beta$ is responsible for the linear birefringence. When $\beta=0$ there is no birefringence, the medium is isotropic, and the pulse evolution is deter- 
mined completely by the nonlinear terms. In this case Eqs. (13) take a simple form given by

$$
\begin{aligned}
& \frac{d}{d \xi} S_{0}=0, \\
& \frac{d}{d \xi} S_{1}=2 g S_{2} S_{3}, \\
& \frac{d}{d \xi} S_{2}=-2 g S_{1} S_{3}, \\
& \frac{d}{d \xi} S_{3}=0 .
\end{aligned}
$$

$S_{0}$ and $S_{3}$ are therefore conserved and the pulse evolution appears as a pure rotation of the Stokes vector around the $e_{3}$ axis onto the Poincare sphere. The solution to Eqs. (20) is

$$
\begin{aligned}
& S_{1}=S_{0} \cos \theta \cos [\omega \xi+\phi / 2], \\
& S_{2}=-S_{0} \cos \theta \sin [\omega \xi+\phi / 2], \\
& S_{3}=S_{0} \sin \theta,
\end{aligned}
$$

where the frequency $\omega=2 g S_{3}=2 g S_{0} \sin \theta, \theta$ is the angle formed between $\mathbf{S}$ and the plane $\left(S_{1}, S_{2}\right)$, which is constant, and $\phi$ defines the initial phase of this rotation. The direction of the rotation depends on the sign of $S_{3}$. This direction is different in each hemisphere. If $\beta=0$ then the two linearly polarized solitons given by Eqs. (7) and (8) coincide. This means that the approximation of the constant profile is a good one in this case. Depending on the initial conditions, the rotation around the $e_{3}$ axis takes place along one of the circles parallel to the equator onto the Poincaré sphere. The state of polarization always remains elliptic. The field components along the axes $u$ and $v$ oscillate periodically. The phase difference between the two components oscillates around $\pi / 2$ (for $S_{3}>0$ ) or around $-\pi / 2$ (for $S_{3}<0$ ). Equation (21) can be described as a solution with oscillating phase.

Note that the phase only oscillates in the laboratory frame of reference connected with the axes $(u, v)$. It is easy to show using Eqs. (21) that, in a frame rotated with the angular frequency $\omega / 2=g S_{0} \sin \theta$ relative to these axes, the state of polarization is fixed (elliptic) [23]. Hence, in the laboratory frame, a nonlinear rotation of the polarization ellipse occurs. The ellipticity is defined by the angle $\theta$ (given by the initial conditions). The polarization changes from linear at $\theta=0$ to circular at $\theta= \pm \pi / 2$. The angular frequency of rotation at a given ellipticity is defined by $g$, i.e., by the energy of the soliton. The direction of rotation is clockwise for the righthanded elliptic polarization [Fig. 2(a)] and counterclockwise for the left-handed polarization [Fig. 2(b)]. The ellipse of polarization does not rotate at $\theta=\pi / 2$ (linear polarization) and/or at small $g$ as should be the case in the limit of small intensities.

The solutions to Eqs. (6) cannot be found in analytic form for the general case but they can in particular cases. Equations (21) for $\theta=\pi / 2$ or $-\pi / 2$ correspond to stationary points $\mathbf{S}=\left(0,0, \pm S_{0}\right)$ on the Poincare sphere, when the circularly polarized input wave does not change its state of polarization, with the two components $u$ and $v$


FIG. 2. Rotation of the ellipse of polarization in an isotropic $(\beta=0)$ nonlinear fiber. (a) $0<\theta<\pi / 2$. (b) $-\pi / 2<\theta<0$.

being $\pi / 2$ out of phase. In terms of $u$ and $v$, these solutions can be written

$$
\begin{aligned}
& u=\frac{\sqrt{q}}{\sqrt{A} \cosh (\sqrt{2 q} \tau)}, \quad v=+i u \text { at } \theta=\pi / 2, \\
& u=\frac{\sqrt{q}}{\sqrt{A} \cosh (\sqrt{2 q} \tau)}, \quad v=-i u \text { at } \theta=-\pi / 2 .
\end{aligned}
$$

Another set of stationary points is located on the equator of the Poincare sphere $(\theta=0)$ at $\mathrm{S}=\left(S_{1}, S_{2}, 0\right)$. The solution for this case is

$u=\frac{\sqrt{2 q}}{\cosh (\sqrt{2 q} \tau)} \cos \frac{\phi}{2}, \quad v=\frac{\sqrt{2 q}}{\cosh (\sqrt{2 q} \tau)} \sin \frac{\phi}{2}$,

where $\phi$ is an arbitrary phase. This solution corresponds to a wave linearly polarized in a direction which forms an angle $\phi / 2$ with the fast axis.

(b) The parameter $A$ is related to the nonlinear birefringence (or ratio between the self- and cross-phase modulation terms). This ratio is zero when $A=1$. In this case Eqs. (13) become

$$
\begin{aligned}
& \frac{d}{d \xi} S_{0}=0, \\
& \frac{d}{d \xi} S_{1}=0, \\
& \frac{d}{d \xi} S_{2}=2 \beta S_{3}, \\
& \frac{d}{d \xi} S_{3}=-2 \beta S_{2} .
\end{aligned}
$$

Therefore $S_{0}$ and $S_{1}$ are conserved and the Stokes vector $\mathbf{S}$ now rotates around the $\mathbf{e}_{3}$ axis with frequency $2 \beta$. The solution to Eqs. (25) is:

$$
\begin{aligned}
& S_{1}=S_{0} \cos \theta^{\prime}, \\
& S_{2}=S_{0} \sin \theta^{\prime} \sin \left(2 \beta \xi+2 \phi_{0}\right), \\
& S_{3}=S_{0} \sin \theta^{\prime} \cos \left(2 \beta \xi+2 \phi_{0}\right),
\end{aligned}
$$

where $\theta^{\prime}$ is the angle formed between $\mathbf{S}$ and $\mathbf{e}_{1}$, and $\phi_{0}$ is another constant to be determined from the initial conditions. The solution for $u$ and $v$ is given by

$$
\begin{aligned}
& u=\frac{\sqrt{2 q} \cos \left(\theta^{\prime} / 2\right)}{\cosh (\sqrt{2 q} \tau)} \exp \left(i \beta \xi+i \phi_{0}\right), \\
& v=\frac{\sqrt{2 q} \sin \left(\theta^{\prime} / 2\right)}{\cosh (\sqrt{2 q} \tau)} \exp \left(-i \beta \xi-i \phi_{0}\right),
\end{aligned}
$$

where $\theta^{\prime}$ defines the relative values of the two components and $2 \phi_{0}$ denotes the phase difference between 
them. The solution oscillates with frequency $2 \beta$. The state of polarization oscillates from linear (when $\beta \xi+\phi_{0}=N \pi / 2, N$ being an integer) to elliptical [when $\left.\beta \xi+\phi_{0}=\left(N+\frac{1}{2}\right) \pi / 2\right]$, and vice versa. The amplitudes of the two components do not change in this case, but their phase difference increases linearly. This means that the phase of the whole solution rotates. Accordingly, this solution can be called a solution with rotating phase.

The differential Stokes parameters are given by

$$
\begin{aligned}
& s_{0}=f(\tau), \\
& s_{1}=f(\tau) \cos \theta^{\prime}, \\
& s_{2}=f(\tau) \sin \theta^{\prime} \cos \left(2 \beta \xi+2 \phi_{0}\right), \\
& s_{3}=f(\tau) \sin \theta^{\prime} \sin \left(2 \beta \xi+2 \phi_{0}\right),
\end{aligned}
$$

where $f(\tau)=2 \sqrt{2 q} / \cosh (\sqrt{2 q} \tau)$. The integrated Stokes parameters become

$$
\begin{aligned}
& S_{0}=1, \\
& S_{1}=\cos \theta^{\prime}, \\
& S_{2}=\sin \theta^{\prime} \cos \left(2 \beta \xi+2 \phi_{0}\right), \\
& S_{3}=\sin \theta^{\prime} \sin \left(2 \beta \xi+2 \phi_{0}\right) .
\end{aligned}
$$

By comparing Eqs. (28) and (29), one realizes that the differential (at $\tau=0$ ) and the integrated Stokes parameters are proportional to each other if the pulse exactly conserves its profile during propagation.

The two examples given above show qualitatively the role of each parameter in Eqs. (6) on the evolution of solitonlike pulses. These two cases correspond to two different regimes of propagation of solitonlike pulses. The qualitative peculiarities of these two regimes are preserved in more complicated cases which we consider in the next section. In general, these two regimes of propagation are defined not only by the parameters of the problem but also by the initial conditions, i.e., by the initial state of polarization.

\section{B. General case}

If neither $\beta$ nor $(1-A)$ is zero, then the total motion consists of a combination of two rotations, as Eqs. (13) or (16) show. To analyze this complicated motion, we first find its singular points on the Poincare sphere. When $S_{0}<\beta / g$, Eqs. (13) have only two stationary points [see Fig. 3(a)]

$$
\begin{array}{lll}
S_{1}=+S_{0}^{\circ}, & S_{2}=0, & S_{3}=0, \\
S_{1}=-S_{0}, & S_{2}=0, & S_{3}=0 .
\end{array}
$$

The point $\mathbf{S}=\left(+S_{0}, 0,0\right)$ is always stable. It corresponds to the slow linearly polarized pulse of Eq. (7). The second point $S=\left(-S_{0}, 0,0\right)$ is stable if $\beta / g>S_{0}$. It corresponds to the fast linearly polarized soliton given by Eq. (8).

If $\beta / g<S_{0}$ then, in addition to (30) and (31), we find two more stationary points [see Fig. 3(b)],

$$
S_{1}=-\frac{\beta}{g}, \quad S_{2}=0, \quad S_{3}= \pm\left(S_{0}^{2}-\frac{\beta^{2}}{g^{2}}\right)^{1 / 2} \text {, }
$$

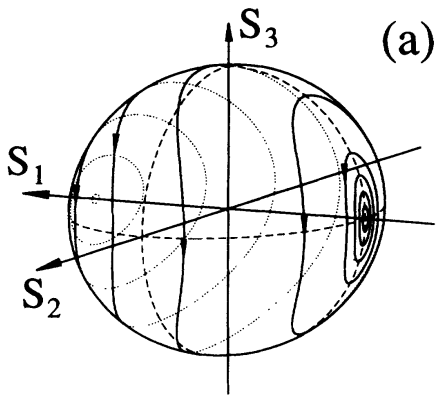

(a)

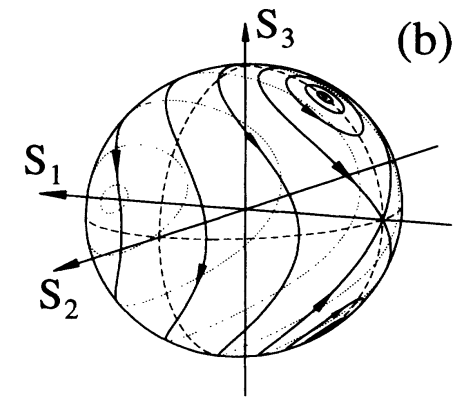

FIG. 3. Trajectories on the Poincare sphere for the periodic solutions for (a) $\beta>g S_{0}$ and (b) $\beta<g S_{0}$.

which are always stable. These two stationary solutions correspond to elliptically polarized solitons with

$$
|X|^{2}=\frac{\sqrt{S_{0}-\beta / g}}{2},|Y|^{2}=\frac{\sqrt{S_{0}-\beta / g}}{2}+\frac{\beta}{g},
$$

and ellipticity $|X|^{2}-|Y|^{2}=-\beta / g$. The phase difference between the two components $u$ and $v$ is $\pi / 2$ or $-\pi / 2$. These two points correspond to right- and left-hand elliptically polarized pulses which change neither their state of polarization nor the amplitudes of their components during the propagation. With the appearance of these two points, the stationary point corresponding to the fast soliton (31) loses its stability. However, the energy $Q$ of these stationary solutions is higher than that corresponding to the fast solitons. This indicates that they can also be unstable. In fact, our numerical simulations verify this unstable behavior.

When points (32) exist, there are solutions oscillating around them. We call them solutions with oscillating phase.

\section{Solutions with oscillating phase}

These solutions correspond to closed loops inside two separatrices in Fig. 3(b). They can be written as

$$
\begin{aligned}
& S_{1}=\frac{A_{1}^{2} k^{2}}{4 \beta g}\left[2 \mathrm{cn}^{2}\left(A_{1} \xi, k\right)-1\right]-\frac{\beta}{g}, \\
& S_{2}= \pm \frac{A_{1}^{2} k^{2}}{2 \beta g} \operatorname{sn}\left(A_{1} \xi, k\right) \operatorname{cn}\left(A_{1} \xi, k\right), \\
& S_{3}= \pm \frac{A_{1}}{g} \operatorname{dn}\left(A_{1} \xi, k\right),
\end{aligned}
$$


where

$$
A_{1}=\frac{2 \beta}{k^{2}}\left[\left(4\left(1-k^{2}\right)+\frac{g^{2} k^{4}}{\beta^{2}} S_{0}^{2}\right]^{1 / 2}-\left(2-k^{2}\right)\right]^{1 / 2},
$$

and sn, cn, and dn are elliptic Jacobi functions with $k$ being their amplitude. Plus and minus signs correspond to loops at $S_{3}>0$ and $S_{3}<0$, respectively. These solutions exist only when $\beta / g<S_{0}$. The value of the second integral is $W=\beta / g+\left(A_{1}^{2} / 4 \beta g\right)\left(2-k^{2}\right)$.

The three parameters $k, A_{1}$, and $W$ are related to each other. In principle, $k$ and $W$ can be expressed in terms of $A_{1}$ (or $k$ and $A_{1}$ in terms of $W$ ). However, we found the above formulas to be the most convenient way to relate these values. At $k \rightarrow 0$ these solutions tend to the stationary solution given by Eq. (32). Note that $A_{1} \rightarrow \sqrt{g^{2} S_{0}^{2}-\beta^{2}}$ at $k \rightarrow 0$. Hence, the solution (34) oscillates in this limit with frequency $2 \sqrt{g^{2} S_{0}^{2}-\beta^{2}}$. The frequency decreases to zero when $k$ increases to 1 . At $k \rightarrow 1$ these solutions degenerate into separatrix solutions which are written down below.

The state of polarization is elliptic with both components oscillating along the principal axes, with the phase difference between them oscillating around $\pi / 2$ or $-\pi / 2$. This regime of propagation is analogous to the regime with oscillating phase considered before. Moreover, at $\beta \rightarrow 0$ these solutions have the limit considered in the previous section. Solutions with oscillating phase can be excited if the initial state of polarization is elliptic, with the major axis of the ellipse directed along the principal axis of the fiber $\left(S_{2}=0\right)$ and with the amplitudes satisfying the inequality $S_{1}<S_{0}-2 \beta / g$ (or $\left.|X|^{2}<|Y|^{2}+S_{0}-2 \beta / g\right)$.

When $\beta \neq 0$, for the same values of material parameters a second type of solution appears. It characterizes a different regime of propagation. We shall call these solutions with rotating phase.

\section{Solutions with rotating phase}

These solutions correspond to closed loops beyond two separatrices in Fig. 3(b). In the case $\beta / g<S_{0}$ they are given by

$$
\begin{aligned}
& S_{1}=\frac{A_{2}^{2}}{4 \beta g}\left[2 d n^{2}\left(A_{2} \xi, k_{1}\right)-1\right]-\frac{\beta}{g}, \\
& S_{2}=\frac{k_{1} A_{2}^{2}}{2 \beta g} \operatorname{sn}\left(A_{2} \xi, k_{1}\right) \operatorname{dn}\left(A_{2} \xi, k_{1}\right), \\
& S_{3}=\frac{k_{1} A_{2}}{g} \operatorname{cn}\left(A_{2} \xi, k_{1}\right),
\end{aligned}
$$

where

$$
A_{2}=2 \beta\left[\left(1-2 k_{1}^{2}\right)+\left[\frac{g^{2}}{\beta^{2}} S_{0}^{2}-4 k_{1}^{2}\left(1-k_{1}^{2}\right)\right]^{1 / 2}\right]^{1 / 2}
$$

and modulus $k_{1}$ satisfies $0<k_{1}<1$. The second integral is given by $W=\beta / g+\left(A_{2}^{2} / 2 \beta g\right)\left(k_{1}^{2}-\frac{1}{2}\right)$. Their corresponding trajectories rotate around the axis $\mathbf{e}_{1}$, with a fre- quency which goes from zero (when $k_{1}=1$ ) to $2 \beta \sqrt{(g / \beta) S_{0}+1}>2 \beta$ (when $k_{1}=0$ ).

If $\beta / g>S_{0}$ then the solution is defined by the same expression (35) but with

$$
A_{2}=2 \beta\left[\left(1-2 k_{1}^{2}\right) \pm\left(\frac{g^{2}}{\beta^{2}} S_{0}^{2}-4 k_{1}^{2}\left(1-k_{1}^{2}\right)\right]^{1 / 2}\right]^{1 / 2},
$$

and the amplitude changes in the smaller interval $0<k_{1}^{2}<\frac{1}{2}-\sqrt{1 / 4-g^{2} S_{0}^{2} / 4 \beta^{2}}$. There are two values for $A_{2}$ (corresponding to the plus and minus signs in front of the second square root) for each value of $k_{1}$. They coincide at $k_{1}^{2}=\frac{1}{2}-\sqrt{\frac{1}{4}-g^{2} S_{0}^{2} / 4 \beta^{2}}$.

When $k_{1} \rightarrow 0$ the solution (35) can be approximated by the formulas

$$
\begin{aligned}
& S_{1}=S_{0}-k_{1}^{2} \frac{2 \beta^{2}}{g S_{0}} \sqrt{1+g S_{0} / \beta}, \\
& S_{2}=\frac{k_{1} A_{3}^{2}}{2 \beta g} \sin A_{3} \xi, \quad S_{3}=\frac{k_{1} A_{3}}{g} \cos A_{3} \xi,
\end{aligned}
$$

where $A_{3}=2 \beta \sqrt{(g / \beta) S_{0}+1}$. In this limit the second integral becomes $W=-S_{0}$. This solution corresponds to rotations around the stable point (30). The solution (36) shows that the point (30) is an elliptic point and that the slow soliton is stable. Note that the frequency of rotation is $\sqrt{(g / \beta) S_{0}+1}$ times higher than $2 \beta$. This means that the difference in propagation constants between the two components in this limit does not coincide with the difference in the linear limit, as was the case of the solution (27). The frequency of oscillations in (35) changes from zero at $k_{1}=1$ to $A_{3}$ at $k_{1}=0$. Therefore, there is some value of $k_{1}$ when the difference in propagation constants is exactly $2 \beta$. The frequency of phase rotation changes from zero (when $k_{1}=0$ ) to $A_{3}$ when $k_{1}=0$. Hence there is a unique value of $k_{1}$ when this frequency is exactly equal to $2 \beta$.

The state of polarization for these solutions evolves as in the case $(A=1)$ of the previous section, except that the amplitudes of the two components along the principal axes oscillate with $\xi$. This changes the parameters of the ellipse of polarization during propagation. The period of the oscillations is also different, and is defined by the period of the Jacobi elliptic functions in (35).

The solutions with rotating phase can be excited in an optical fiber in two simple ways. First, any linearly polarized soliton $\left(S_{3}=0\right)$ can be used as an input (except the one which coincides with the fast soliton). Secondly, we can use an elliptically polarized soliton with the major axis of the polarization ellipse directed along the principal axis of birefringence $\left(S_{2}=0\right)$. The amplitudes of two components must, in this case, satisfy the inequality $S_{1}>S_{0}-2 \beta / g$ (or $|X|^{2}>|Y|^{2}+S_{0}-2 \beta / g$ ).

\section{Separatrix solution}

The solution (34) at $k \rightarrow 1$ and the solution (35) at $k_{1} \rightarrow 1$ have a common limit in the form of the separatrix solution 


$$
\begin{aligned}
& S_{1}=\frac{\delta^{2}}{4 \beta g}\left[\frac{2}{\cosh ^{2}(\delta \xi)}-1\right]-\frac{\beta}{g}, \\
& S_{2}= \pm \frac{\delta^{2}}{2 \beta g} \frac{\sinh (\delta \xi)}{\cosh ^{2}(\delta \xi)}, \quad S_{3}= \pm \frac{\delta}{g \cosh (\delta \xi)},
\end{aligned}
$$

where $\delta=\sqrt{4 \beta\left[g S_{0}-\beta\right]}$. The evolution parameter $W=S_{0}$ for this solution. At $\xi \rightarrow-\infty$ the trajectory corresponding to this solution tends to the point $\mathbf{S}=\left(-S_{0}, 0,0\right)$. The Stokes vector moves away from this point exponentially when $\xi$ increases. Hence in the vicinity of this point the solution can be approximated by

$$
\begin{aligned}
& S_{1}=-S_{0}, \\
& S_{2}= \pm \frac{\delta^{2}}{\beta g} \exp (\delta \xi), \quad S_{3}= \pm \frac{2 \delta}{g} \exp (\delta \xi) .
\end{aligned}
$$

The Stokes vector moves exponentially to the same point when $\xi \rightarrow \infty$. Hence, close to this point, the solution can be approximated by

$$
\begin{aligned}
& S_{1}=-S_{0}, \quad S_{2}=\mp \frac{\delta^{2}}{\beta g} \exp (-\delta \xi), \\
& S_{3}= \pm \frac{2 \delta}{g} \exp (-\delta \xi) .
\end{aligned}
$$

Two trajectories start at the point $S=\left(-S_{0} 0,0\right)$ and two trajectories finish at this point. Hence, it is a saddle-type point. The separatrices are shown in Fig. 3(b) as two closed loops starting and finishing at the point $\mathbf{S}=\left(-S_{0} 0,0\right)$.

\section{Stability of the fast solitons}

The expressions (38) and (39) show that, when $\beta / g<S_{0}$, the point $S=\left(-S_{0} 0,0\right)$ is of saddle type and that fast solitons are unstable. Obviously, the value $\delta$ is the growth rate of this instability. Let us estimate it by calculating the value of $g$. The point $S=\left(-S_{0}, 0,0\right)$ corresponds to the fast linearly polarized soliton (8). If it is used as the initial condition, then $X(\xi)=0$ and $Y(\xi)=1$. For a quartz fiber $A=\frac{2}{3}$, and

$$
g=\frac{4}{9}(q+\beta) .
$$

Hence, the point $S=\left(-S_{0}, 0,0\right)$ is unstable if $g<\beta$ or $q>\frac{5}{4} \beta$. In the opposite case, $g>\beta$ or $q<\frac{5}{4} \beta$, it is stable. The instability growth rate is equal to

$$
\delta=\frac{4}{3} \sqrt{\beta\left(q-\frac{5}{4} \beta\right)} .
$$

This expression describes qualitatively the curve plotted in Fig. 1(b). The slight shift in the edge of the stability region $[q / \beta=1.53$ rather than 1.25 given by Eq. (41)] is due to the approximations we made.

In addition to this instability, we have numerically found instability with a complex growth rate in the interval $-0.2<q / \beta<1.475$. Only the real part of the growth rate is shown in Fig. 1(b). This instability is related to the oscillations of the pulse shape during propagation, and radiation due to these oscillations. It cannot be described using our simple approximation. In the small range $1.475<q / \beta<1.53$ the frequency of the oscillations is very close to zero. The radiation is small in this case and the fast soliton is stable.

\section{The role of the radiation}

All the above solutions were obtained analytically in the approximation of constant shape. Of course, the shape is not fixed but oscillates slightly. This implies that a small amount of radiation is emitted in each period of these oscillations. We have observed that the emission of radiation is low when $\beta / g \ll<S_{0}$ and high for values of $\beta / g$ larger than $S_{0}$. This means that, if we start from a point corresponding to an unstable fast mode, its subsequent trajectory will never come back to this point but will tend to reduce the total energy of the pulse. More precisely, the trajectory evolves in such a way that the total energy $Q$ reduces its magnitude as well as that of the second integral $W$. In the above theory we can take the radiation into account by considering that the values of $Q$ and $W$ adiabatically decrease with $\xi$. Then the solutions would behave qualitatively as described above, but in such a way that trajectories in Fig. 3 slowly slide from one closed loop to another one with slightly smaller value of $W$.

On the energy-dispersion diagram [Fig. 1(a)], for a fixed $\beta$ we associate a given energy $Q$ to a soliton state with a specified propagation constant $q$. At any given $q$ the energy can change from the value for the slow soliton, $Q_{s}=2 \sqrt{2(q-\beta)}$, to the value for the fast soliton, $Q_{f}=2 \sqrt{2(q+\beta)}$. On the other hand, the second integral $W$ is equal to $-S_{0}$ for the slow soliton and $+S_{0}$ for the fast soliton. At any fixed $q$, the second integral monotonically decreases from the upper curve to the lower one. In our simple analysis we can consider the second integral as being roughly linearly proportional to the energy $Q$ along the vertical line. Then the energy of the solitonlike pulse is a function of two variables,

$$
\begin{aligned}
Q(q, W)= & \frac{1}{2}\left[\left(S_{0}+W\right) 2 \sqrt{2(q+\beta)}\right. \\
& \left.+\left(S_{0}-W\right) 2 \sqrt{2(q-\beta)}\right] .
\end{aligned}
$$

Any energy losses from a given solitonlike pulse can be associated with a decrease of $q$ and $Q$.

Obviously, in the presence of radiation, a representative point in Fig. 1(a) can move only downwards, decreasing $W$. For an infinite length of propagation the result of this tendency will be the convergence of any initial condition to a slow mode because the slow mode has the lowest energy at given $q$. From our numerical simulations, we have found that the rate of this process depends on $\beta / g$ and is negligible for $\beta / g \ll 1$ (or $q / \beta \rightarrow \infty$ ). It becomes considerable when $\beta$ and $g$ are comparable.

As a consequence, a trajectory starting at any point of the Poincare sphere will tend, on the average, to reduce its $W$ value. If this process is slow, the trajectories will slide from one closed loop in Fig. 3 to another one with smaller $W$. The decrease of $W$ gradually changes the type of solutions. This produces a change in the type of evolution of the polarization state of the pulse. In particular, transitions from solutions with oscillating phase to 
solutions with rotating phase are possible.

The decrease of $q$ at given $W$ is only connected with the slow reshaping of the average profile of the pulse. In general, both $q$ and $W$ decrease due to radiative processes and a representative point on the energy-dispersion diagram moves down as well as to the left along the parabolic curves. This behavior is what we qualitatively observed in our numerical simulations.

\section{NUMERICAL SIMULATIONS}

We have numerically solved Eqs. (6) rather than the equations for the Stokes parameters by using a split-step method. We divide the equation into a dispersive part and a birefringent and nonlinear part. At each step we solve the dispersive part using the fast Fourier transform. The rest of the equation is solved using a fourth-order Runge-Kutta method. The temporal grid was typically chosen to have 8192 points to cover the interval $\left[-\tau_{\max }, \tau_{\max }\right]$, where $\tau_{\max }$ is much larger than the width of the initial input. The step size in the $\xi$ direction was typically taken to be $\Delta \xi=0.01$. Numerical accuracy was checked by repeating the simulations for different grid and step sizes. To remove the small amplitude waves emitted from the soliton we used reflectionless absorbers at the end points of the temporal grid. In all simulations we used the value $A=\frac{2}{3}$. The Stokes parameters are calculated from the values of $u$ and $v$.

The definition of the Stokes parameters given by Eqs. (14) requires knowledge of the pulse amplitudes. In the numerical simulations it is more convenient to use the integrated Stokes parameters defined in the form

$$
S_{i}=\left[\int_{-\tau_{M}}^{\tau_{M}} s_{i} d \tau\right] / S_{0}(\xi=0),
$$

where the integration must cover the whole pulse but nothing else, to eliminate the influence of the radiation around the pulse. For cases when the shape does not change, $S_{i}$ in this definition is equal to $S_{i}$ defined by (14). Normalization in (43) is chosen to satisfy $S_{0}(\xi=0)=1$ (at least initially). If the shapes change significantly, the definition (43) is preferable because the length of the Stokes vector is proportional to the energy of the pulse, and this changes more smoothly than the pulse amplitudes. In cases where the pulse shape changes by a small amount, the integrated Stokes parameters are roughly proportional to the differential Stokes parameters at $\tau=0$ and we can represent data using $s_{i}(\tau=0)$ rather than $S_{i}$. Obviously, the trajectories presented through any of these three definitions exhibit the same qualitative behavior.

First we studied the unstable behavior of the fast linearly polarized waves given by Eq. (8). In terms of the Stokes parameters, we start from the point $\mathbf{S}=\left(-S_{0}, 0,0\right)$. This point is unstable inside the intervals we discussed before, and any perturbation like the addition of a small value $u=\mu v$ to the stationary solution makes it diverge from the initial point. Depending on whether the small parameter $\mu$ is real or imaginary, the solutions with oscillating or rotating phase will be excited.
Figure 4(a) shows the evolution of the Stokes parameters for $\beta=0.01, q=1$, and $\mu=10^{-4} i$. The trajectory evidently is very close to the separatrix. However, the perturbation is chosen in such a way that the solution with rotating phase is excited. Hence, after moving along the first separatrix with $S_{3}>0$ and returning to the saddletype point, it follows the second separatrix with $S_{3}<0$. The trajectory would follow the same separatrix if the real value $\mu=10^{-4}$ were used. The deviation of the differential Stokes parameter $s_{0}(\tau=0)$ at $\tau=0$ from its initial value is less than $2 \%$ during this evolution. This indicates that the pulse shape changes very slightly, as we have verified by direct observation of the profiles, and that our approximation is good enough for this simulation. Radiation emission is also very small, and consequently the trajectory is described by our approximation with very high accuracy. Figure 4(b) shows the evolution of the field amplitudes at the origin for the two components.

Perspective plots of the field amplitudes $|u|$ and $|v|$ for this simulation are shown in Fig. 5. This figure shows that the energy is concentrated mainly in the fast mode
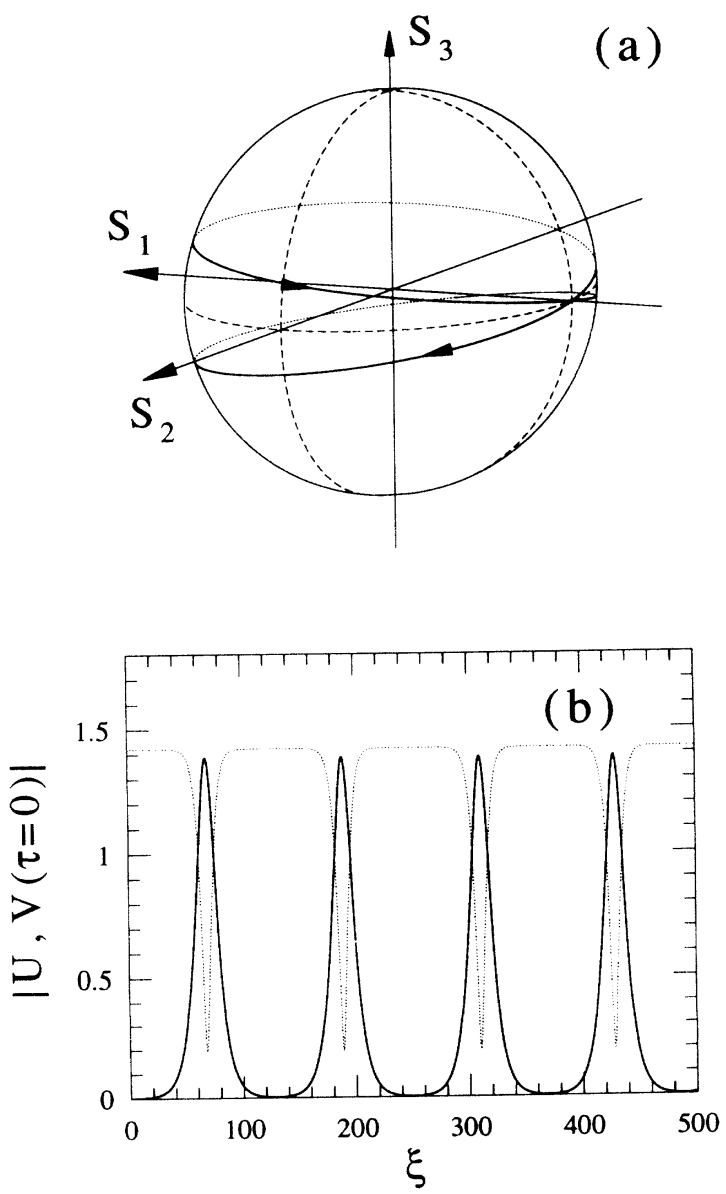

FIG. 4. (a) Evolution of the integrated Stokes parameters for $\beta / q=0.01$. The initial condition is the fast linearly polarized wave [Eq. (8)] with $q=1$. A periodic solution close to the separatrix is excited. The solution still has oscillating phase. (b) Evolution of pulse amplitudes $|u|$ and $|v|$ at the center of pulses $(\tau=0)$. 



FIG. 5. Perspective plot for the field envelopes $|u|$ and $|v|$ for the same parameters as in Fig. 4.

and that it is certainly unstable, so that, after some distance of propagation, the energy switches to the slow mode. Because of the recurrency which takes place for separatrices, the energy switches back to the fast mode where it stays for a longer distance. Absolute values of the fields are plotted in Fig. 5. Therefore, the plot does not show whether the first or the second regime of propagation takes place. The distance of propagation is $\xi=500$ in this case. The behavior is the same (almost periodic) for longer distances of propagation. The periodicity becomes weaker for larger values of $\beta / q$.

Figure 6(a) shows the evolution of the Stokes parameters for $\beta=0.04$ and $q=1$. The evolution is qualitatively the same as in the previous case, except that the total energy, $S_{0}$, and the parameter $W$ slowly decrease due to the radiation effects. The trajectory gradually shifts away from the separatrix, becoming a periodic solution with rotating phase. The period of the oscillations eventually decreases. At a distance $\xi=500$ we can observe nine periods of the cn function. Radiation is still small in this case and the total energy of the pulse decreases only by $0.3 \%$ over the distance $\xi=500$, as we can see in Fig. $6(\mathrm{~b})$, where the total energy defined by Eq. (2) is plotted.

Figure 7(a) shows the evolution of the Stokes parameters for $\beta=0.1$ and $q=1$ and for the initial conditions associated with the corresponding fast mode. We can see that increasing $\beta / q$ causes more radiative losses. The parameter $W$ as well as $S_{0}$ decreases on average. The trajectory qualitatively corresponds to the solution with rotating phase. Because the initial input is unstable, energy is shared between each component and after some transition distance the amplitudes become approximately equal on average, but oscillate periodically and thus exchange the energy. The total pulse energy decreases by $5 \%$ over the distance $\xi=300$. The evolution of $W$ is shown in Fig. 7(b).

An example of the solution with oscillating phase is given in Fig. 8. We now consider $\beta=0.01$ and take as in-
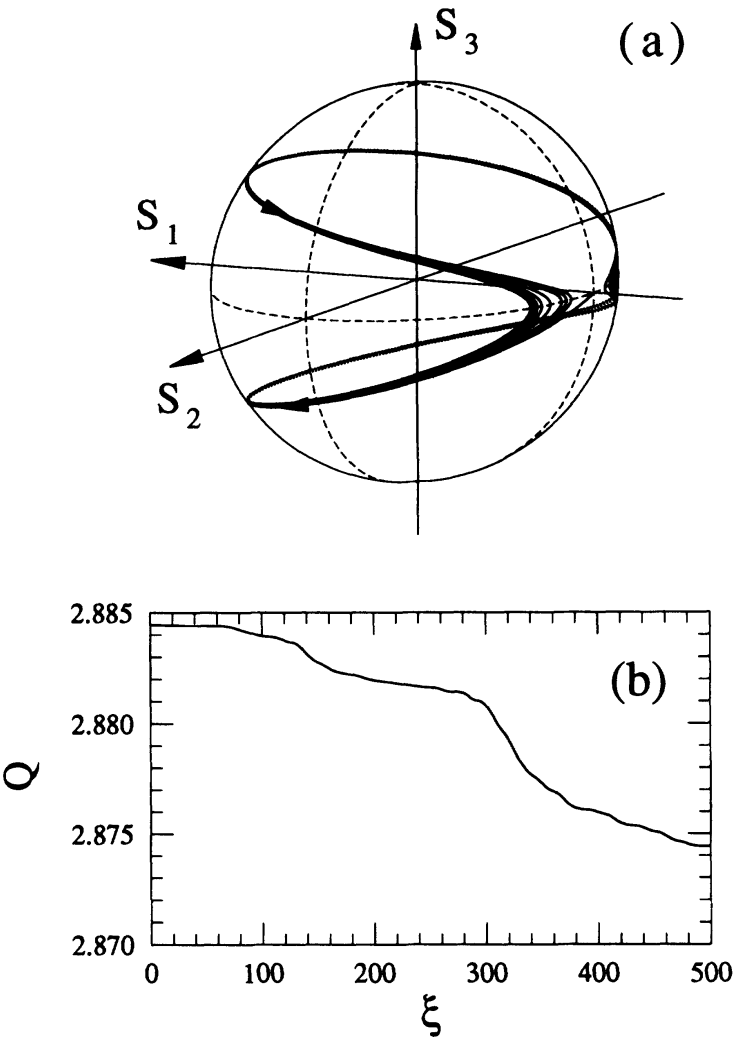

FIG. 6. (a) Evolution of the integrated Stokes parameters for $\beta / q=0.04$. The initial condition is the unstable fixed point $\mathbf{S}=\left(-S_{0}, 0,0\right)$ with a small perturbation. (b) Evolution of the total energy in this simulation.

itial conditions the following:

$$
\begin{aligned}
& u=\sqrt{2(q+\beta)} \operatorname{sech}[\sqrt{2(q+\beta)} \tau] \sin (\pi / 16), \\
& v=i \sqrt{2(q+\beta)} \operatorname{sech}[\sqrt{2(q+\beta)} \tau] \cos (\pi / 16),
\end{aligned}
$$

with $q=1$. This initial condition corresponds very roughly to the input value of a solution with oscillating phase. In fact, the trajectory converges to the solution very quickly in a propagation distance $\xi<\approx 1$. The evolution parameter $W$ for this solution is higher than $S_{0}$, which shows that the energy is higher than for the separatrix. Therefore radiation can, in principle, transform the solution with oscillating phase through the separatrix to the solution with rotating phase. This can happen when the radiation is considerable.

Figure 9 shows this type of transformation. Now $\beta=0.1$ and the initial condition is

$$
\begin{aligned}
& u=\sqrt{2(q+\beta)} \operatorname{sech}[\sqrt{2(q+\beta)} \tau] \sin (\pi / 12), \\
& v=i \sqrt{2(q+\beta)} \operatorname{sech}[\sqrt{2(q+\beta)} \tau] \cos (\pi / 12),
\end{aligned}
$$

where $q=1$. The trajectory starts almost at the stationary point, makes several loops corresponding to the solution with oscillating phase and then transforms into a solution with rotating phase. This trajectory ends up converging to the slow soliton. Its radius in the Poincaré space decreases, which means that a large amount of radiation is emitted in this process. In Fig. 9 we have plot- 

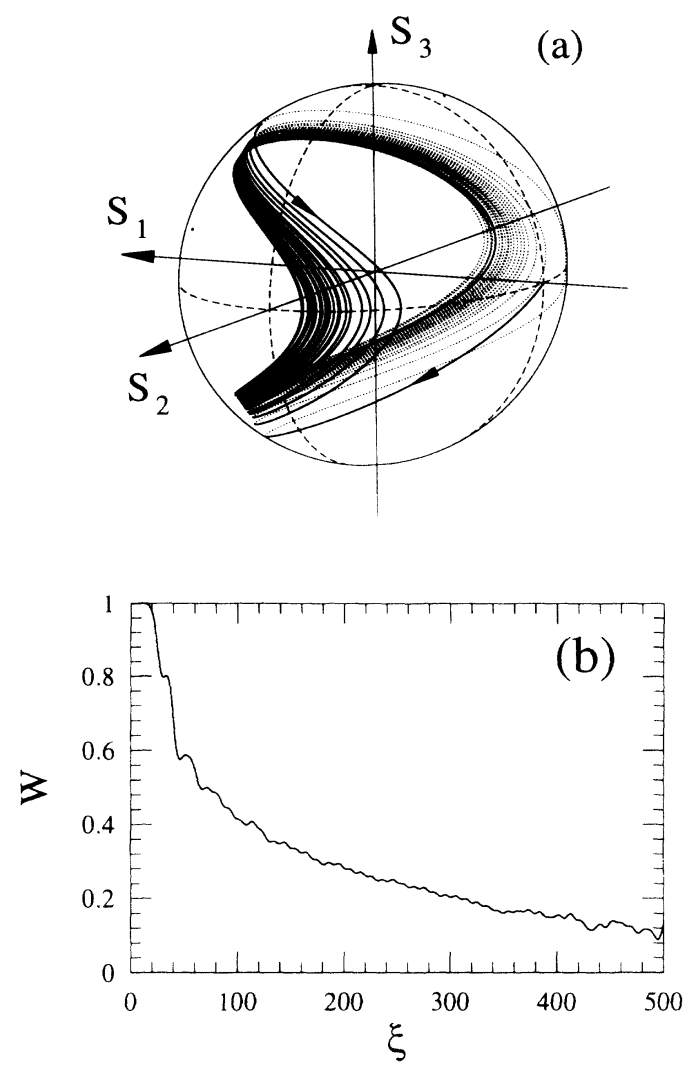

FIG. 7. (a) Evolution of the integrated Stokes parameters for $\beta / q=0.1$. The initial condition is chosen to excite the solution with rotating phase. The total pulse energy decreases due to radiative losses. (b) Evolution of $W$.

ted only three parts of the total trajectory representing the stages (a) $0<\xi<100$, (b) $400<\xi<500$, and (c) $900<\xi<1000$.

When $q / \beta<1.475$, only solutions with rotating phase can exist. To illustrate the general behavior we observed in this region, Fig. 10(a) shows the evolution of the Stokes parameters for $\beta=2$ and initial conditions corresponding to a fast soliton for $q=1$. In this region, the instability is of radiative type. The leading pulse decreases its energy



FIG. 8. Evolution of the integrated Stokes parameters for $\beta / q=0.01$. The initial condition is chosen to excite the solution with oscillating phase.

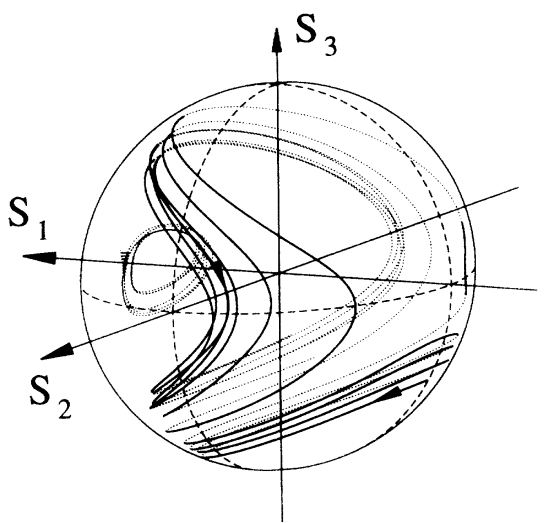

FIG. 9. Evolution of the integrated Stokes parameters for $\beta / q=0.1$. The initial condition is chosen to excite the solution with oscillating phase.

on propagation, and therefore $S_{0}$ decreases and the Poincaré sphere shrinks in size. The trajectory rotates around the $S_{1}$ axis along surfaces with constant $W$. The value of $W$ decreases adiabatically [Fig. 10(b)]. This decrease is negligible initially when the amplitude of oscillations is small and radiation is also small. The decrease becomes faster in the region $60<\xi<100$ when the amplitude of oscillations increases. The decrease becomes noticeably slower later on $\left(S_{1}>0\right)$ when the oscillations of the am-
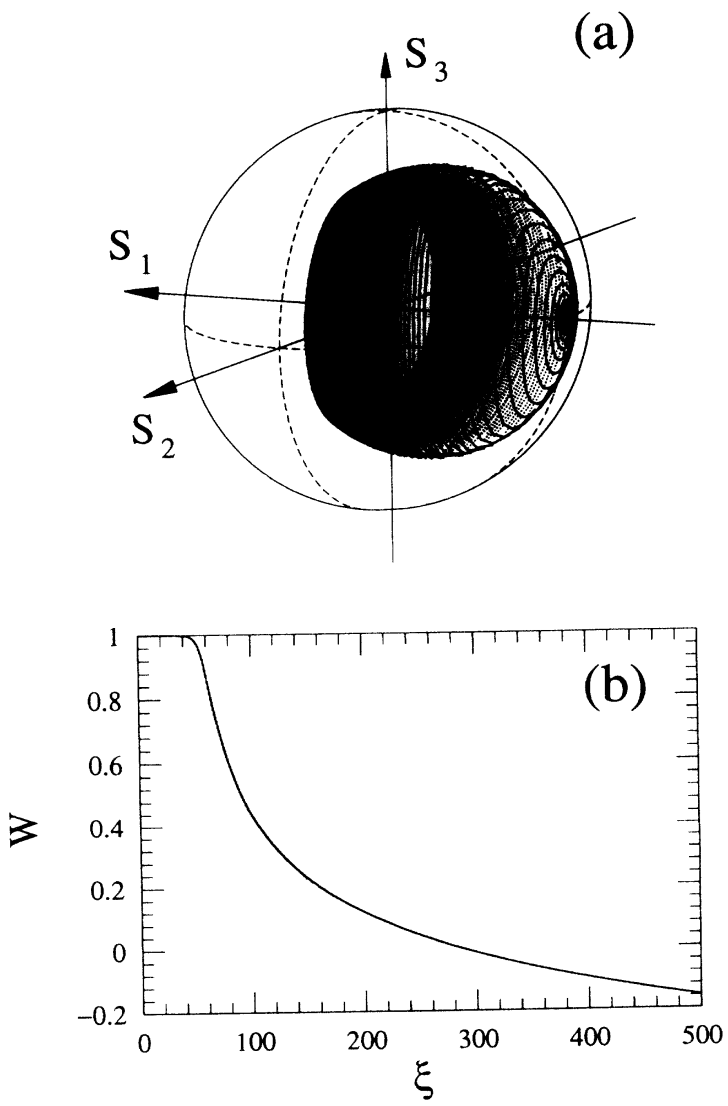

FIG. 10. (a) Evolution of the integrated Stokes parameters for $\beta=2, q=1$. The initial condition is the corresponding fast soliton. (b) Evolution of $W$. 

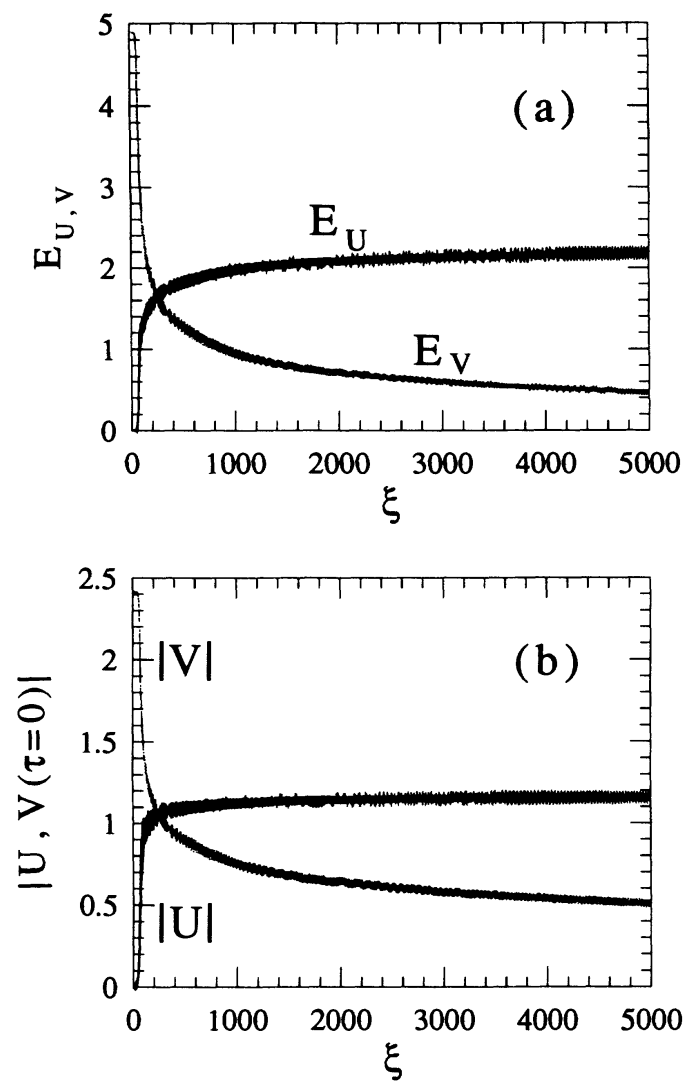

FIG. 11. Evolution of (a) the energies in each of the two components and (b) their field amplitudes at $\tau=0$ as a function of $\xi$ for $\beta=2, q=1$.

plitudes of the fast and the slow components become smaller. This is the reason why the trajectory lines are denser above the equatorial line $S_{1}=0$. However, the total energy decreases continuously due to the radiation process, and the radius of the Poincare sphere decreases.

We continued the simulations up to $\xi=5000$. Only a part of these simulations (up to $\xi=500$ ) is shown in Fig. 10. Figure 11(a) shows the distribution of the energy between the two components, and Fig. 11(b) shows the evolution of their field amplitudes. These figures show that the solution converges to the slow soliton at a very slow rate. The soliton parameter also changes on propagation. The starting value of $q / \beta$ is 0.5 . This ratio becomes 0.47 when $\xi$ is around 250 and becomes 0.33 when $\xi=5000$. In this particular example the soliton parameter $q$ decreases.

The above simulations generally confirm the qualitative picture we outlined in the previous section.

\section{DISCUSSION}

Now we shall concentrate on the main points of this investigation.

(1) The problem of nonlinear pulse propagation in optical birefringent fibers defines a Hamiltonian dynamical system with an infinite number of degrees of freedom. This dynamical system can be considered as the one defined by the NLSE plus a small perturbation. There- fore the solitonlike solutions of this system are perturbed solitons of the single NLSE. An approximation of an average profile can be used to solve the problem. The average profile is close to the soliton of the NLSE but does not necessarily coincide with it. Moreover, the solution usually oscillates around this average profile.

(2) A solitonlike pulse of average profile can be represented by the point (representative point) on the energy-dispersion diagram which specifies its energy $Q$ versus the soliton parameter $q$. The stationary solutions of this dynamical system (the slow and the fast solitons) are represented by points on two parabolic curves on the diagram. In general, the representative points for solitonlike pulses are located between these two curves. Slow (adiabatic) changes of the pulse in the course of propagation along the fiber can be represented by the slow motion of the representative point on the energy-dispersion diagram.

(3) The state of polarization is the physical parameter in this problem which can be directly measured (rather than the average profile). Its evolution can be conveniently represented by the Stokes parameters. The approximation of the average profile allows us to reduce the integrodifferential equations for the differential Stokes parameters to a simple set of ordinary differential equations for the integrated Stokes parameters of the soliton as a whole. This approximation greatly simplifies the analysis and allows us to represent the solutions in the form of trajectories on the Poincaré sphere. The analytic solutions of this set describe the evolution of a solitonlike input pulse with great accuracy when the linear beat length $L_{l}$ between the two linearly polarized components is much larger than the nonlinear beat length.

(4) The approximation of the average profile allows us to find simple analytic expression for the nonlinear beat length $L_{\mathrm{nl}}$, in terms of the soliton parameter and the material parameters. It also allows us to define an evolution parameter $W$, which serves to characterize the type of solution. The analysis shows that, depending on the value of $W$, two qualitatively different regimes of evolution of the state of polarization are possible. They are characterized by the form taken by the phase difference between the two field components on propagation. We call them "solutions with oscillating phase" and "solutions with rotating phase." Initial conditions needed for the excitation of these two regimes of propagation in optical fibers have been considered.

(5) When the two beat lengths are comparable, one has to take into account the losses of energy from the leading pulse due to the radiation processes, as they are important in this case. To understand the effects of the radiation emission, our qualitative analysis on the Poincare sphere must be done bearing in mind the slow motion of the representative point on the energy-dispersion diagram. A decrease of energy in the leading pulse associated with an adiabatic decrease of the evolution parameter $W$ (the representative point moves vertically on the energy-dispersion diagram) changes the regime of evolution of the state of polarization. In particular, the transformation from the solution with oscillating phase to the solution with rotating phase is possible in this case. The 
decrease of energy in the leading pulse associated with an adiabatic diminution of the soliton parameter $q$ (the representative point in the dispersion diagram moves along the curve $Q$ vs $q$ for the NLSE soliton) changes the average soliton profile. The evolution parameter $W$ decreases faster than the soliton parameter $q$. As a result the solution converges to the slow soliton after long distances of propagation.

\section{CONCLUSIONS}

In conclusion, we have shown that the evolution of the state of polarization in birefringent optical fibers can be qualitatively described using the equations for the integrated Stokes parameters. Different regimes of propagation were found, depending on the fiber parameters and on the initial conditions. We have linked the Stokesparameter formalism on the Poincare sphere with the analysis using the energy-dispersion diagram. It was shown that the radiation effects can be naturally included into this combined qualitative analysis. The results of our analytical approach were confirmed by exact numerical simulations.

\section{ACKNOWLEDGMENTS}

The work of N.A. is supported by the Australian Photonics Cooperative Research Centre (APCRC). N.A. thanks Professor A. W. Snyder for stimulating discussions. The work of J.M.S.-C. was supported by the ClCyT under Contract No. T1C91-0361. The authors are grateful to Dr. Adrian Ankiewicz for helpful discussions and for the critical reading of this manuscript.
[1] H. G. Winful, Appl. Phys. Lett. 47, 213 (1985).

[2] K. J. Blow, N. J. Doran, and D. Wood, Opt. Lett. 12, 202 (1987).

[3] S. G. Evangelides, L. F. Mollenauer, J. P. Gordon, and N. S. Bergano, J. Lightwave Technol. 10, 28 (1992).

[4] C. R. Menyuk, Opt. Lett. 12, 614 (1987).

[5] E. M. Wright, G. I. Stegeman, and S. Wabnitz, Phys. Rev. A 40, 4455 (1989).

[6] C. R. Menyuk, IEEE J. Quantum Electron. QE-25, 2674 (1989).

[7] D. N. Cristodoulides and R. I. Joseph, Opt. Lett. 13, 53 (1988).

[8] S. Trillo, S. Wabnitz, E. M. Wright, and G. I. Stegeman, Opt. Commun. 70, 166 (1989).

[9] M. Eguchi, K. Hayata, and M. Koshiba, Opt. Lett. 16, 82 (1991).

[10] P. A. Bélanger and C. Paré, Phys. Rev. A 41, 5254 (1990).

[11] B. Daino, G. Gregory, and S. Wabnitz, J. Appl. Phys. 58, 4512 (1985).

[12] C. R. Menyuk, J. Opt. Soc. Am. B 10, 1585 (1993).

[13] P. K. A. Wai, H. H. Chen, and Y. C. Lee, Phys. Rev. A
41, 426 (1990).

[14] Asymptotics Beyond All Orders, edited by H. Segur et al. (Plenum, New York, 1991).

[15] J. Satsuma and N. Yajima, Prog. Theor. Phys. Suppl. No. 55, 284 (1974).

[16] A. L. Berkhoer and V. E. Zakharov, Zh. Eksp. Teor. Fiz. 58, p. 903 (1970) [Sov. Phys. JETP 31, 486 (1970)].

[17] Y. Inoue, J. Plasma Phys. 16, 439 (1976).

[18] S. V. Manakov, Zh. Eksp. Teor. Fiz. 65, 505 (1973) [Sov. Phys. JETP 38, 248 (1974)].

[19] L. D. Faddeev and L. A. Takhtajan, Hamiltonian Methods in the Theory of Solitons (Springer-Verlag, Berlin, 1986).

[20] M. Romagnoli, S. Trillo, S. Wabnitz, Opt. Quantum Electron. 24, S1237 (1992).

[21] N. N. Akhmediev and A. Ankiewicz, Phys. Rev. Lett. 70, 2395 (1993).

[22] J. M. Soto-Crespo and N. N. Akhmediev, Phys. Rev. E 48, 4710 (1993).

[23] M. Born and E. Wolf, Principles of Optics (Pergamon, New York, 1959), Sec. 1.4. 

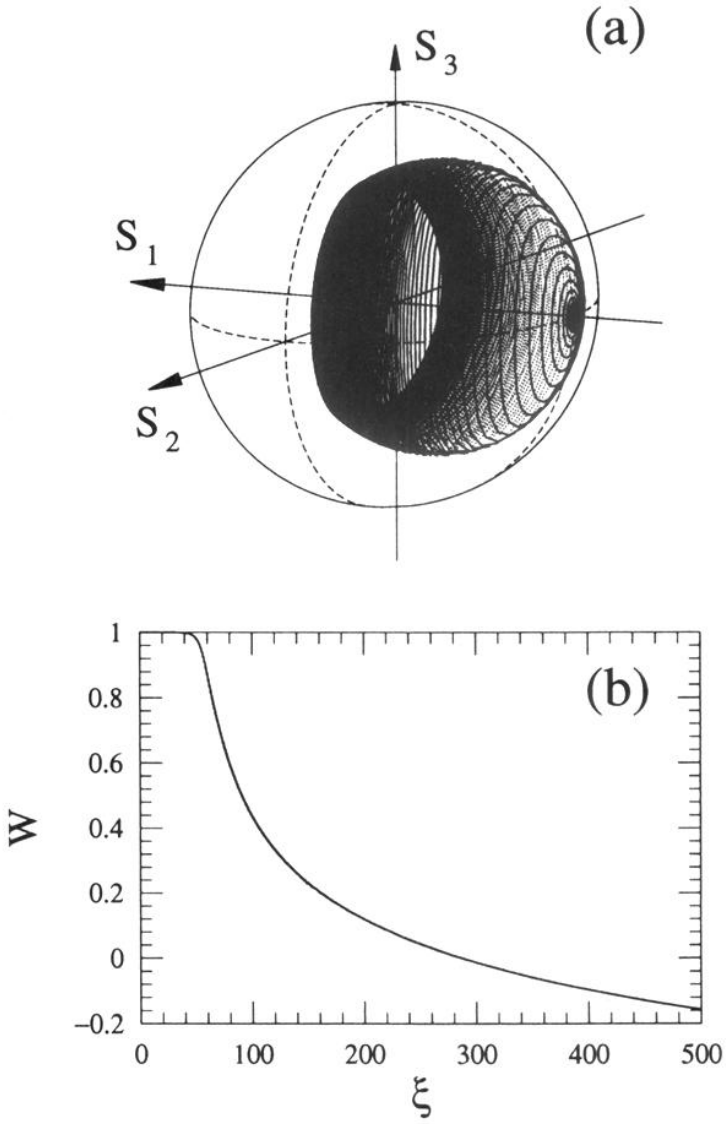

FIG. 10. (a) Evolution of the integrated Stokes parameters for $\beta=2, q=1$. The initial condition is the corresponding fast soliton. (b) Evolution of $W$. 